\title{
Estructura y propiedades de la alinita
y del cemento de alinita $(*)$
}

B. Nocdeliax, Prof br. en Ciencias

I. BIKBAOC, Candidato a Dr. en Ciencias

A. Svextsitski, Candidato a Dr. en Ciencias, Instituto de Investigaciones Cientificas y proyectos de Materiales de Construcción, Tachkent $y$

v. ILtKHINe, Dr. en Ciencias, Instituto de Cristalografia de la Icademia de la C.R.s.s.

7. Congreso Internacional de la Química de los Cementos. Paris, 1980; pp. v-169 v-1 il

Uno de los compuestos más importantes del clínker de cemento obtenido, según la tecnología de baja temperatura ("tecnología de sal"), es la alinita, nuevo silicato cálcico que cristaliza en el sistema cúbico. La célula elemental, de parámetros: $a=10,471 ; c=8,614 \AA$ contiene nueve átomos independientes: $3 \mathrm{Ca}, 1 \mathrm{Si}, 4 \mathrm{O}$ y $1 \mathrm{Cl}$. La fórmula puede representarse como sigue:

$$
\mathrm{Ca}_{11}(\mathrm{Si}, \mathrm{Al})_{4} \mathrm{O}_{18} \mathrm{Cl}
$$

La densidad calculada es $3,10 \mathrm{~g} / \mathrm{cm}^{3}$, mientras que su valor experimental es $3,075 \mathrm{~g} / \mathrm{cm}^{3}$. Por difracción de rayos $\mathrm{X}$ se comprobó que existen tres posiciones de cationes de calcio (de gran tamaño), cristalográficamente diferentes con diferencias también en el entorno.

La posición de los átomos de cloro en la estructura cristalina, característica de la alinita, es particularmente importante; se encuentran rodeados por ocho átomos de calcio, lo que le confiere determinadas características y propiedades.

El espectro infrarrojo de la alinita se interpreta por las vibraciones de valencia y de deformación de los grupos $\mathrm{SiO}_{4}$ y $\mathrm{AlO}_{4}$ (ver tabla en la pág. siguiente).

A partir de cristales pulverizados se han obtenido espectros ESR de la alinita con radioespectrómetro RE-1301, en $3 \mathrm{~cm}$; la irradiación de los cristales del mineral a 298 y $77^{\circ} \mathrm{K}$ se efectuó en un aparato PXM-20 con una dosis de $10^{19}$ roentgens. El espectro resultante es diferente al correspondiente a la alita.

(*) La indudable importancia de la "Fabricación de Cemento a Baja Temperatura (Tecnologia-NTS)" patentada por los Científicos de la URSS, aconsejan que la Revista MATERIALES DE CONSTRUCCION ofrezca un resumen del trabajo presentado en París, en el 7." Congreso de la Química de los Cementos, por el Prof. NOUDELMAN y Colaboradores.

El presente resumen y la correspondiente traducción han sido realizados por Olga Fernández-Peña y Tomás Vázquez, del IETCC.

El trabajo original contieno 7 figuras, 2 tablas (reproducidas en este resumen) y 4 referencias bibliográficas, entre las cuales está la siguiente: "Bulletin des découvertes et des inventions (1979), diplôme n." 210 , n." 22 , p. 3 (russe)". 
Identificación de las bandas de absorción infrarroja de la alinita

\begin{tabular}{|c|c|c|c|c|c|c|c|c|c|c|c|c|c|c|}
\hline \multicolumn{15}{|c|}{ Clasificación de las bandas de absorción (números de onda, en $\mathrm{cm}^{-1}$ ) } \\
\hline \multicolumn{6}{|c|}{ Bandas de valencia } & \multicolumn{9}{|c|}{ Bandas de deformación } \\
\hline \multicolumn{3}{|c|}{$\mathrm{SiO}_{4}$} & & \multicolumn{2}{|c|}{$\mathrm{Al}_{2} \mathrm{O}_{3}$} & \multicolumn{2}{|c|}{$\mathrm{SiO}_{4}$} & \multicolumn{7}{|c|}{$\mathrm{SiO}_{4}\left(\delta_{\mathrm{s}}\right)$} \\
\hline \multicolumn{3}{|c|}{$\nu_{a s}$} & $\nu_{8}$ & & as & & & \multicolumn{7}{|c|}{$y: \mathrm{AlO}_{4}\left(\delta_{\mathrm{as}}, \delta_{\mathrm{s}}\right)$} \\
\hline 936 & 928 & 918 & 837 & 760 & 730 & 560 & 500 & 385 & 370 & 353 & 333 & 324 & 309 & \\
\hline 902 & 896 & & & & & 430 & 398 & 295 & 281 & 270 & 250 & 231 & 221 & 208 \\
\hline
\end{tabular}

La composición química de la alinita en el clínker varía, dentro de límites estrechos, en función de la cantidad de elementos que puede llevar: $\mathrm{Al}, \mathrm{Fe}, \mathrm{Mg}, \mathrm{Cl}$ y otros contenidos en las materias primas. Cristaliza en el clínker en forma de prismas, de placas hexagonales y de agujas que son frecuentemente restos de cristales desfigurados.

Las dimensiones de los cristales de la alinita en diferentes clínkeres pueden variar dentro de límites bastante amplios, desde fracciones hasta cientos de micras. La microscopía óptica permite observar la estructura fina, media y gruesa, en gránulos de clínker de alinita. Se observa que la cristalización de este compuesto no es siempre similar; en la superficie de los cristales aparecen en ocasiones maclas polisintéticas. Los estudios del clínker efectuados con microscopía electrónica permiten determinar claramente el tipo de cristalización de la alinita. Su índice de refracción tiene un valor 1,709 $\pm 0,001$. En función de la naturaleza y cantidad de elementos que contiene, puede elevarse hasta 1,714-1,715. Los autores del presente trabajo han realizado estudios sobre solubilidades de elementos que pueden entrar a formar parte de la alinita policristalina, con diversas concentraciones de óxidos de $\mathrm{Al}, \mathrm{Mg}, \mathrm{Fe}, \mathrm{K}, \mathrm{Na}, \mathrm{P}$ y de $\mathrm{B}$.

La cocción de la alinita con esas mezclas y el análisis de las fases de los productos de cocción a través de la difracción de rayos $\mathrm{X}$, han permitido determinar los límites de solubilidad siguientes (\% en peso); $\mathrm{Al}_{2} \mathrm{O}_{3}=4,5 ; \mathrm{Fe}_{2} \mathrm{O}_{3}=3,0 ; \mathrm{MgO}=3,0 ; \mathrm{BaO}=2,5$; $\mathrm{K}_{2} \mathrm{O}, \mathrm{Na}_{2} \mathrm{O}$ menos de 0,$5 ; \mathrm{P}_{2} \mathrm{O}_{5}, \mathrm{~B}_{2} \mathrm{O}_{3}=1$. Estos valores pueden variar en $\pm 10 \%$; en caso de presencia múltiple de esos elementos puede haber una desviación del valor dado de $\pm 20 \%$.

La temperatura de fusión de la alinita varía dentro de límites comprendidos entre 1.420$1.490^{\circ} \mathrm{C}$, en función de la composición química y de los elementos que contiene. El elevado número de elementos que pueden entrar en la red cristalina del compuesto, unido a la elevada simetría de la estructura y de su "friabilidad", explica las oscilaciones de la temperatura de fusión. El límite superior corresponde a la alinita con un mínimo de impurezas.

Los autores han realizado estudios de alinita a altas temperaturas lo que es de gran interés para el conocimiento de la química-física del compuesto. Estos estudios los han llevado a cabo por difracción de rayos $\mathrm{X}$. Las variaciones de los valores de las líneas de difracción con el aumento de temperatura, ha permitido confeccionar 4 series principales de líneas de difracción con intensidad variable.

Se ha comprobado la reversibilidad del proceso en caso de recalentamiento o reenfriamiento de la alinita, lo que demuestra que las cuatro series corresponden a otras tantas 
modificaciones polimórficas: 3 de las series correspondientes a alta temperatura $\left(\beta, \alpha^{\prime}\right.$ y $\left.\alpha\right)$ son de simetría cúbica. En el siguiente cuadro se dan los datos de la difracción de rayos $\mathrm{X}$ de la alinita.

Datos de la difractometría de rayos $X$ de las modificaciones de la alinita

\begin{tabular}{|c|c|c|c|c|c|c|c|c|c|c|c|}
\hline \multicolumn{3}{|c|}{$\begin{array}{l}\text { Alinita a temperatura } \\
\text { ambiente }(\gamma)\end{array}$} & \multicolumn{3}{|c|}{$1.050^{\circ} \mathrm{C}(\beta)$} & \multicolumn{3}{|c|}{$1.190^{\circ} \mathrm{C} \quad\left(\alpha^{\prime}\right)$} & \multicolumn{3}{|c|}{$1.190^{\circ} \mathrm{C}(a)$} \\
\hline \multicolumn{3}{|c|}{$\begin{array}{l}\text { Célula tetragonal } \\
\text { (centrada en el cuerpo) } \\
\mathrm{a}=10,4714 \pm 0,0019 \AA\end{array}$} & \multicolumn{3}{|c|}{$\begin{array}{c}\text { Célula cúbica } \\
\text { (centrada en las caras) } \\
\mathrm{a}=7,865 \pm 0,00 \AA\end{array}$} & \multicolumn{3}{|c|}{$\begin{array}{c}\text { Célula cúbica } \\
\text { (centrada en el cuerpo) } \\
\mathrm{a}=11,82 \pm 0,2 \AA\end{array}$} & \multicolumn{3}{|c|}{$\begin{array}{c}\text { Célula cúbica } \\
\text { (centrada en las caras) } \\
\mathrm{a}=8,060 \pm 0,005 \AA\end{array}$} \\
\hline hkl & d & 1 & hkl & d & 1 & hkl & d & 1 & hkl & d & 1 \\
\hline 031 & 3,235 & 22 & 111 & 4,54 & 22 & 211 & 4,82 & 44 & 111 & 4,65 & 22 \\
\hline 222 & 2,808 & 100 & 200 & 3,93 & 27 & 222 & 3,41 & 2 & 200 & 4,03 & 32 \\
\hline 040 & 2,618 & 26 & 220 & 2,784 & 100 & 321 & 3,154 & 100 & 220 & 2,850 & 100 \\
\hline 141 & 2,436 & 9 & 311 & 2,370 & 12 & 330 & 2,782 & 40 & 311 & 2,430 & 9 \\
\hline 240 & 2,342 & 5 & 222 & 2,272 & 5 & 420 & 2,647 & 18 & 222 & 2,326 & 2 \\
\hline 042 & 2,237 & 4 & 400 & 1,966 & 38 & 422 & 2,414 & 6 & 400 & 2,015 & 34 \\
\hline 033 & 2,218 & 10 & 331 & 1,802 & 4 & 431 & 2,322 & 9 & 331 & 1,850 & 7 \\
\hline 004 & 2,154 & 20 & 420 & 1,758 & 7 & 440 & 2,086 & 9 & 420 & 1,802 & 14 \\
\hline 510 & 2,054 & 5 & 422 & 1,607 & 23 & 530 & 2,026 & 4 & 422 & 1,646 & 42 \\
\hline 341 & 2,035 & 5 & 511 & 1,512 & 4 & 600 & 1,971 & 25 & 511 & 1,552 & 6 \\
\hline 143 & 1,903 & 8 & 440 & 1,391 & 7 & 611 & 1,918 & 2 & 440 & 1,425 & 7 \\
\hline 440 & 1,851 & 19 & & & & 620 & 1,871 & 9 & & & \\
\hline 350 & 1,796 & 2 & & & & 631 & 1,743 & 3 & & & \\
\hline 600 & 1,745 & 5 & & & & 550 & 1,670 & 4 & & & \\
\hline 053 & 1,692 & 12 & & & & 552 & 1,610 & 4 & & & \\
\hline 352 & 1,658 & 12 & & & & 642 & 1,580 & 9 & & & \\
\hline 253 & 1,610 & 10 & & & & & & & & & \\
\hline 035 & 1,545 & 15 & & & & & & & & & \\
\hline 071 & 1,474 & 6 & & & & & & & & & \\
\hline
\end{tabular}

El clínker de alinita utilizado para la producción de cemento contiene, gereralmente, del 60 al $70 \%$ de alinita. No obstante para el cemento de endurecimiento rápido y para el de alta resistencia inicial, se utiliza clínker hasta con el $80 \%$. El resto del clínker contiene ortosilicato, aluminatos y ferritos de cloruro de calcio. La proporción de estas fases no tiene unos límites concretos, ya que están condicionados por el tipo de crudos y el destino a que se aplique el cemento.

La elevada actividad en la hidratación de los cementos de alinita se explica por las particularidades de su estructura (presencia de átomos de $\mathrm{Cl}$ "activos" en la estructura del nuevo silicato y un gran número de defectos en la red cristalina), por la composición de la fase líquida (débil contenido de álcalis, presencia de $\mathrm{Cl}^{-}$, concentraciones elevadas de $\mathrm{Ca}^{2+}$ ), por la composición y la estructura de los nuevos compuestos hidratados (formación sobre la superficie de la alinita de una envoltura hidratada metaestable, permeable y relativamente bien cristalizada). Estas propiedades logran una hidratación profunda y el endurecimiento de ese tipo de cemento a temperaturas ambientes, e incluso por debajo de cero (hasta $-5^{\circ} \mathrm{C}$ ) y facilidad para el tratamiento térmico y húmedo.

Dado que el clínker de alinita posee una alta porosidad y una elevada aptitud a la molienda, es posible intensificar la hidratación y el endurecimiento de los cementos aumen- 
tando su finura. Así, por ejemplo, para una superficie específica de cemento portland de $3.000 \mathrm{~cm}^{2} / \mathrm{g}$ la del cemento de alinita sería de $4.500 \mathrm{~cm}^{2} / \mathrm{g}$, con consumos de energía eléctrica idénticos (aproximadamente $30 \mathrm{~kW} / \mathrm{h}$ ). Hay que destacar que, a diferencia del cemento portland, tal aumento de la superficie específica del cementơ de alinita no exige una cantidad de agua mayor, sino, por el contrario, menor. Los valores mínimos de la relación agua/cemento son 0,225 a 0,235 con una superficie específica de $4.500 \pm 200 \mathrm{~cm}^{2} / \mathrm{g}$. El sulfato cálcico añadido en forma de yeso, de fosfoyeso o de boroyeso, es un compuesto necesario, pero sin jugar el papel de retardador de fraguado que realiza en el caso del cemento portland; al contrario: es un acelerador del endurecimiento en el cemento de alinita.

El contenido óptimo del yeso en este cemento varía entre límites de 2 a $3,5 \%$ (calculado como $\mathrm{SO}_{3}$ ). Con ello se consigue sobrepasar la velocidad de hidratación media de la alita en 1,5 a 2,5 veces. Hay un endurecimiento rápido del cemento de alinita a edades cortas. Además, el endurecimiento del cemento no finaliza con la hidratación de la alinita. La explicación que los autores dan a este fenòmeno se basa en que los silicatos hidratados primarios, del tipo $\mathrm{C}_{2} \mathrm{SH}_{2}$ recristalizan en silicatos hidratados secundarios $\mathrm{CSH}$. El proceso va seguido: por el de la cristalización del $\mathrm{Ca}(\mathrm{OH})_{2}$; por el del aumento del grado de polimerización de los hidrosilicatos; por el de las deformaciones de retracción; por el de la reducción del contenido en agua de cristalización y por el del aumento del contenido en silicatos hidratados. Los autores continúan considerando la posibilidad de formación de hidrosilicatos de cloruro cálcico durante la hidratación y endurecimiento de la alinita. El contenido de iones $\mathrm{Cl}^{-}$en el cemento endurecido a edades avanzadas está dentro, general. mente, de los límites del 0,3 al $0,4 \%$.

La permeabilidad del cemento endurecido es de 5 a 10 veces menor que la del cemento portland, lo que garantiza — según los autores - una estabilidad alta de los hormigones armados.

La resistencia de los cementos a base de alinita es del 2 al $30 \%$ superior a la alcanzada por los cementos portland, a igual dosificación de cemento. Otra gran ventaja que los autores de este trabajo destacan, es el gran aumento de las resistencias de los hormigones tratados al vapor: a los 28 días el aumento de resistencias de las probetas tratadas con vapor es del 15 al $25 \%$ superior con respecto a las probetas curadas en condiciones normales. Estos estudios han contribuido a obtener cementos de alta resistencia y hormigones con $600-700 \mathrm{kp} / \mathrm{cm}^{2}$.

Las CONCLUSIONES que los autores del trabajo resaltan, son en resumen:

La alinita es un nuevo silicato tricálcico con cloro en su estructura. Este ion es muy estable al encontrarse rodeado por ocho átomos de $\mathrm{Ca}$, lo que condiciona las características especiales de la alinita: formación a $1.000-1.100^{\circ} \mathrm{C}$, alta simetría de las redes cristalinas de sus modificaciones estructurales, alto grado de solubilidad de elementos modificadores, débil resistencia al molido, alto poder de hidratación, morfología distinta de las formaciones hidratadas. Los autores finalizaron su trabajo diciendo que:

Las cualidades halladas en la alinita y cementos a base de este compuesto, permiten considerar a estos conglomerantes como una alternativa al cemento portland tradicional. 